\title{
Ethics
}

\section{The Marginalization of Chronic Pain Patients on Chronic Opioid Therapy}

John F. Peppin, DO

From: The Pain Treatment Center of the Bluegrass, Lexington, KY.

Dr. Peppin is Director of the Clinical Research Division, The Pain Treatment Center of the Bluegrass, Lexington, KY; Clinical Associate Professor, University of Kentucky, Lexington, $\mathrm{KY}$; and Associate Medical Director, Hospice of the Bluegrass, Lexington, $\mathrm{KY}$

Address correspondence: John F. Peppin, DO, FACP

Director Clinical Research Division The Pain Treatment Center of the Bluegrass 2416 Regency Road Lexington, KY 40503

E-mail: johnpeppin@msn.com

Disclaimer: There was no external funding in the preparation of this manuscript. Conflict of interest: None.

Manuscript received: 01/10/2009 Accepted for publication: 04/09/2009

Free full manuscript: www.painphysicianjournal.com
The realities of treating chronic pain do not reflect the attention that marginalization of patients taking opioids has received. Physicians continue the same prejudices and biases that were present decades ago. One theory proposed to explain this poor treatment has been titled, the "barriers to pain management." The barriers are not treated as moral issues, but rather as clinical aberrations and do not explain continued poor treatment. However, the barriers do not explain certain types of cases where there appears to be specific unfounded concerns related to a specific class of medications, e.g, opioids. Four cases are presented, from the authors experience, illustrating the marginalization of chronic pain patients on chronic opioid therapy admitted to a tertiary care hospital. These types of cases have not been presented in the literature previously and illustrate the failure of the barriers to explain marginalization. In each of these cases mental status changes was the presenting problem. However, in each of these cases, these changes were not related to their opioids, but were explained by clear reasons, other than opioids. Regardless, in each case, the attending physician blamed the opioids, without further workup and stopped them reflexively. It is proposed that there may be more complex psychosocial issues involved in the marginalization of chronic pain patients. This case series illustrates a ubiquitous problem demanding further examination and discussion. It is hoped that this case series will create interest in further research in this area.

Key words: Chronic pain, opioids, marginalization, bias, barriers

Pain Physician 2009; 12:493-498

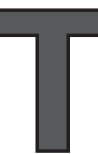

he problem of treating both acute and chronic pain has become such a serious concern that the United States Congress dedicated 2000-2010 as the "Decade of Pain Control and Research" to support pain research and education (1). Unfortunately, media attention has frequently been pejorative and focused on diversion and abuse of scheduled medications rather than reflecting the complexity of successfully treating patients with chronic pain (2). Seventy-five million Americans suffer persistent pain, and many are chronically impaired in daily function (3). Ferrell describes the current status of pain management as "the moral outrage of unrelieved pain"(4). Although the number of pain medicine physicians has increased, Weinstein has shown that many physicians still harbor the same prejudices towards patients taking 
opioid analgesics that were described in the 1970s (5). Thus the treatment of pain with opioid analgesics continues to suffer from over 3 decades of inertia and prejudice. As Rich states, "the silence on the failure of caregivers to adequately address pain in the clinical setting has been deafening" (6). If, as the American College of Physicians Manual of Medical Ethics states, "[T]he primary goals of the physician are to relieve suffering, prevent untimely death, and improve the health of the patient while maintaining the dignity of the person," then the medical profession has not successfully fulfilled its responsibilities (7).

The question that requires analysis is: Why does the health care system continue to provide substandard care of patients in pain? One proposed schema to explain this phenomenon has been called "barriers to pain management" and is listed in Table 1 (8-14).

However, critics of the "barriers" suggest that they do not account for the persistence of poor pain treatment $(15,16)$. If it were as simple as alleviating physicians' concerns about addiction or regulatory sanction, or improving the treatment of pain through education, then pain treatment would have improved dramatically over the past 3 decades. Yet despite the dramatic growth of pain education through the development of professional pain organizations, pain journals, pain conferences, and continuing medical education, poor pain management persists in clinic practice. It seems clear that the barriers are treated merely as clinical aberrations rather than true moral, ethical, or psychosocial issues (6). Since current attempts at explicating the failure of pain care have been unsatis- factory, it is vital to understand why the treatment of pain seems to be so different from the treatment of other medical problems.

As can be seen from the brief discussion above, a central aspect of the treatment of chronic pain patients is the use of opioids and the reaction they generate amongst health care professionals and the public. However, how the health care community reacts to patients who are placed on long-term opioid therapy is not well represented in the medical literature. This paper presents 4 cases that illustrate a pattern of provider reactions to pain patients who were also taking opioids, and offers a discussion of possible causes of the medically inappropriate behavior. This paper posits that the cases described are not isolated but rather manifestations of a more pervasive problem of marginalization. The cases are presented to generate discussion and investigation into the more complex psychosocial issues that are involved in the marginalization of chronic pain patients, that the barriers theory may not take into consideration.

\section{Case Studies}

\section{Case \# 1}

A 68-year-old female presented to the ER for atypical facial pain with concomitant weight loss and depression. She had a history of facial pain for about 20 years. Over the prior 12-18 months she lost over 60 pounds, and became severely malnourished. This was initially related to her major depression. Although she had a long history of depression, it had worsened

Table 1. Potential barriers to effective pain treatment.

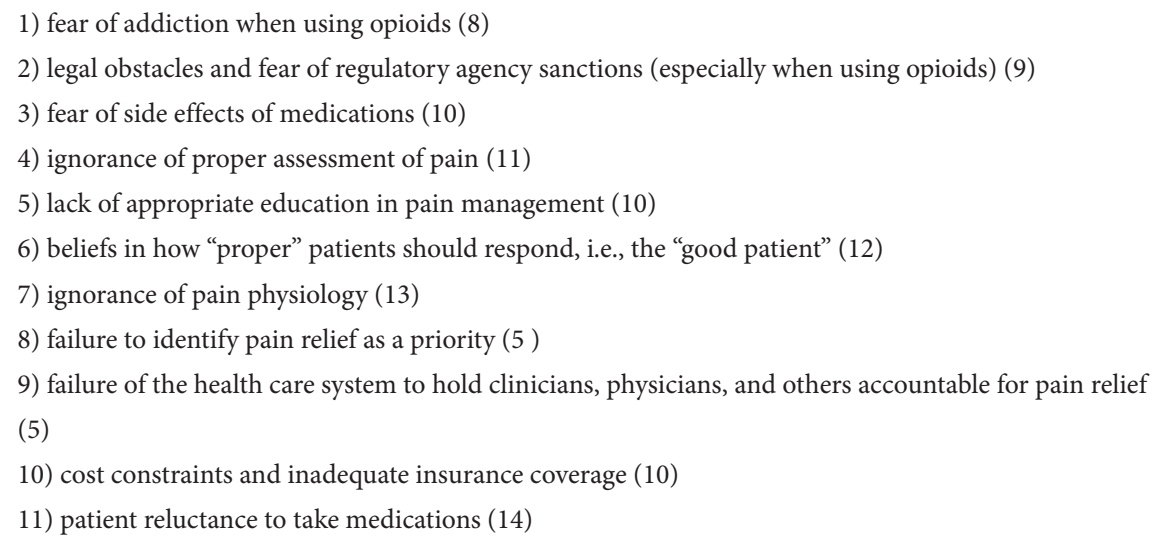


over the preceding months. She was admitted and the pain medicine service was consulted. She was placed on low dose methadone, as well as venlafaxine and gabapentin. Her pain was reduced and she was sent to in-patient rehabilitation to improve her functioning and nutrition. On a late Sunday afternoon she was seen by the pain medicine service and the methadone was increased from $2.5 \mathrm{mg}$ TID to $3 \mathrm{mg}$ TID. The gabapentin was increased from $200 \mathrm{mg}$ to $300 \mathrm{mg}$ TID and the venlafaxine from 75 to $150 \mathrm{mg}$ per day. Two hours later she was found to be unresponsive and was taken to the emergency department and subsequently admitted to ICU. Once consciousness was regained she was delirious and disoriented. She was seen by neurology who stated the episode was due to "narcotics" and "medication overdose." The pain service and internal medicine completed a further workup. She had only received one increased dose of the methadone, $0.5 \mathrm{mg}$, and did not receive increased gabapentin or venlafaxine. These medications were discontinued by neurology; however, the patient failed to improve, and her condition actually worsened. A further workup found an abdominal mass which proved to be pancreatic cancer that had metastasized to the brain. The patient was placed back on low dose methadone and her pain improved although she remained confused and disoriented until she died in hospice.

\section{Case \#2}

A 57-year-old male patient with a history of severe diabetes, diabetic peripheral polyneuropathy, coronary artery disease, peripheral vascular disease, and bilateral below the knee amputations due to non-healing diabetic ulcerations was brought to the ER after being found unresponsive at home. He had a long history of severe nausea and vomiting due to diabetic gastroparesis and had trouble keeping down medications. He also had diabetic diarrhea, which further complicated his medical and pain management. Two years previously, the pain medicine service had started on a subcutaneous infusion of morphine, in the outpatient setting, which worked well for his pain and avoided the gastrointestinal route for obvious reasons. He had been on the subcutaneous infusion for over one year without any problem and at stable doses. His kidney and liver function had been normal. He was transferred to a tertiary care facility for further care. The patient was seen by internal medicine and a diagnosis of "narcotic overdose" was given and his opioid infusion was completely stopped. Three days later the pain medicine service was consulted. The patient was in florid opioid abstinence syndrome with tachycardia, nausea, vomiting, diaphoresis, hypertension, and dramatically increased pain. A review of the chart showed that this patient's blood sugar had been $50 \mathrm{mg} \%$ when he arrived by ambulance to the ER. He had received one amp of Dextrose 50 before being transferred to the tertiary care facility. The patient's sub-cutaneous infusion was restarted and his pain finally came under control. He was alert and oriented at discharge on the dose he had been on before his admission.

\section{Case \#3}

An 82-year-old female with a past history of multiple back surgeries, failed laminectomy syndrome, and chronic severe lower back pain, was admitted through the ER for an exacerbation of severe back pain. The patient was seen by the pain medicine service. She had been placed on a long-acting oxycodone formulation of $40 \mathrm{mg}$ TID by another pain physician in the community. She had been on this dose for well over 2 weeks and brought an almost empty bottle with her. She had also been placed on gabapentin $600 \mathrm{mg}$ TID. She was changed to sustained release 24 -hour morphine at $120 \mathrm{mg}$ per day since she had not been tried on oral morphine before. The next day the patient was somnolent and hard to arouse. The primary service was called and claimed it was "due to the narcotics." The pain medicine service noted that she had normal renal and hepatic function when she entered the hospital, had been on these medications before she was admitted, and that the primary service should reconsider diagnostic impression. Additionally, it was mentioned that her morphine dose was very conservative; since she was opioid tolerant she should have accepted this dose without problem. The primary service ignored the pain service's note. The patient developed hypotension that evening and the primary service was called. The primary service told the nurse to "call the pain people." The pain medicine service gave the patient a fluid bolus and ordered laboratories which showed a BUN/Cr of 57/2.8. The patient was diagnosed with acute tubular necrosis and acute renal failure and was transferred to the ICU where renal failure was treated. She had been placed on celecoxib by the primary service at admission. Two days later her delirium had not improved. Neurology was consulted and said it was "due to the narcotics" which had been stopped 3 days earlier. The pain medicine service signed off the case 
in protest due to the poor medical treatment this patient was receiving. She was subsequently placed on ketorolac and hydrocodone/APAP, even though her renal failure had been due to an NSAID.

\section{Case \#4}

A 58-year-old female patient with peripheral neuropathy due to diabetes, failed laminectomy syndrome, and arthritis presented to the ER with mental status changes. She was seen in the ER and admitted to the hospital with a presumptive diagnosis of "narcotic overdose." The patient was on 4 hydrocodone/ APAP (10/325) tablets per day as well as baclofen 10 mg TID and gabapentin $600 \mathrm{mg}$ TID. She had been on these stable doses for the last 12 months. There were no changes in her kidney or liver functions. Her pain had been well-controlled on these stable doses. She was admitted and all her analgesic medications were stopped. Both the psychiatric service and the pain medicine service were consulted. Pill counts showed no evidence of overuse of her medications and both services stated that her mental status changes were unlikely a result of either her pain or psychiatric medications. A clear cause was never determined in-house, but a fuller workup was left for the pain medicine service to do in the clinic. She was discharged back on her same medications with the unsupported diagnosis of "medication overdose."

A discussion of these types of patients might help in explicating the reasons for continued marginalization of chronic pain patients in general. I use marginalization defined as "...to relegate to an unimportant or powerless position within a society or group"(17). This term illustrates these 4 cases and their subsequent outcomes. It also illustrates the failure of the barriers to explain such marginalization. These cases were taken from a pain medicine hospital service which specializes in the medical management of chronic pain. In each of these cases, patients on chronic opioid therapy had events of altered consciousness which were treated as opioid toxicity purely by impulse, without further workup or evaluation. The opioids were immediately considered the reason for the altered conscientiousness and thoughts of other possible etiologies were abandoned, prima facie. Through a discussion of these 4 cases it can be seen that other issues must be playing a major role in the marginalization these patients received and the poor treatment decisions used by the physicians involved. It is important to realize that this article is not meant as a thorough discussion and evaluation of this topic.

\section{Discussion}

Although this article presents only 4 cases from a single tertiary Midwest metropolitan hospital, this author believes they describe a pervasive and ubiquitous problem of marginalization of a specific group of patients, i.e., chronic pain patients on chronic opioid therapy who are admitted to tertiary care hospitals. The aforementioned definition of marginalization is illustrated in a clinical context by how the 4 cases were approached by the physicians involved. It is my belief that such marginalization is pervasive in the care of chronic pain patients particularly if they are taking opioids. In each of these 4 cases patients on chronic opioid therapy had events of altered consciousness which were treated as opioid toxicity, without further workup or evaluation. The opioids were immediately considered the reason for the altered consciousness and thoughts of other possible etiologies were abandoned, prima facie. Describing similar cases will help to better clarify whether this pattern is widely pervasive.

The 4 cases reveal a pattern of biased clinical reasoning, leading physicians to reflexively attribute changes in mental status to opioids while overlooking other causes. Why opioid medication might act as a magnet for misdiagnosis in cases of mental status changes is important to understand because of the potential impact of this pattern on patient care.

The fear of abuse, addiction, and concern with regulatory oversight should not have entered into the consideration of any of these cases. Each of these patients was psychosocially stable with no aberrant behavior and was followed by a pain specialist who was ultimately responsible for any regulatory issues of misuse or abuse. However, each patient presented with mental status changes in a context where the notion of a "good patient" should have less bearing on management $(18,19)$. All of these patients had insurance coverage for medications and treatment; therefore concern with third party issues should not have been prominent. In a hospital setting, medication administration was carefully monitored for compliance and use. Clearly the patient's mental status changes were the most important issue during these admissions and the assessment should have been no different than for any other case of mental status changes. Although many of other medications have delirium as a poten- 
tial side effect, it was the "narcotic" that was initially attributed as causal (and became a myopic focus), even though clinically the patients had demonstrated tolerance to the CNS effects of opioids. Although fear of side effects could explain not initiating opioid analgesics, it does not explain why opioids become a de facto "cause" for each of these patient's delirium, given that each had been on opioids for months prior to admission.

There was no question that the hospital involved did not hold these physicians accountable. However, in general, "medical staffs" often suffer from the same biases leading to marginalization as the physicians treating the 4 cases above. Green et al (20) have documented that most physicians practicing in the United States have not received appropriate education in the pathophysiology, evaluation, and management of chronic pain conditions in outpatient, acute hospital, and palliative care settings. Apparently in these cases, the workup of mental status change, which should be standard medical practice, was subverted by what must be assumed to be a psychosocial bias leading to erroneous clinical reasoning in the face of data that demonstrate that pain itself and other etiologies are more common causes of delirium than opioids $(21,22)$.

The term "opiophobia" has been used in the literature, but it is not clear why there is a "phobia" against the use of opioids for pain, and this discussion needs to be addressed in more detail. Again, as Rich states, "We should not be surprised if, when closely examined, opioidphobia is complex and multifaceted" (6). It is this complexity and multifacetedness that must be further explored.

There has been little in the literature addressing the rationale behind the marginalization of chronic pain patients on opiate therapy. The "barriers theory" does not explain why these patients were so poorly treated. Health care professionals may respond to those in chronic pain at a deeper, more visceral level that may partially explain marginalization. Thus the lens of psychology may be one way to begin the discussion on this issue (6). Dr. Samuel Perry reflects on the under treatment of burn patients: "...Investigating the under-medication for pain ... revealed a grand irony: the staff's need to preserve a modicum of pain stemmed from the same dynamic that made patients preoccupied with pain, they were all struggling under the most regressive and threatening of circumstances to maintain a coherent sense of self and confirm that they are still alive" (23). Perry additionally states, "...The widespread reluctance among medical professionals to prescribe adequate doses of narcotics also may derive from unconscious factors, including the projected wishes and fears of defying constraints and the need to preserve a modicum of pain to define the sick role, to maintain ego boundaries and to provide reassurance that the patient is alive" (24).

Everyone will have pain. However, the number of hours given to pain and palliative medicine in medical schools does not reflect the pervasiveness of this experience (25). Since pain is universal, and since the goals of medicine are to relieve suffering, it is difficult to understand why professional medical educational organizations have not been more aggressive in addressing these issues. There are data to suggest that changes in medical education can impact medical students' attitudes towards pain treatment and pain patients (26). The Association of American Medical Colleges and other organizations responsible for medical school curriculum and accreditation must take a leadership role in these areas. I argue that medical education needs a twenty-first century review, similar to that of Flexner in 1913 (27). However, even if major changes were to occur today, it would take decades before these would be evaluated in clinical practice. Therefore, investigations into such poor care of chronic pain patients are critically important. Investigations into issues of race and gender have generated understanding of biases and prejudices and have led to improving the prior marginalization of these groups. A similar approach could be applied to reduce bias(es) toward chronic pain patients on opioid therapy.

The above cases are presented in hope of beginning a dialogue that will form the basis of more in-depth discussion and research. A methodologically rigorous approach will be required to define the causes of bias and to develop a rationale for changing practice patterns that will improve the quality of life for chronic pain patients. 


\section{References}

1. Nelson R. Decade of pain control and research gets into gear in USA. Lancet 2003; 362:1129.

2. Raymong J, Underwood A. Taking a new look at pain. Newsweek 2003; May 19, $2003($ CXLI):44-52.

3. Gureje O, Von Korff M, Simon GE, Gater R. Persistent pain and well-being: AWorld Health Organization Study in Primary Care. JAMA 1998; 280:147-151.

4. Ferrell BR. The role of ethics committees in responding to the moral outrage of unrelieved pain. Bioethics Forum 1997; 13:11-16.

5. Weinstein SM, Laux LF, Thornby JI, Lorimor RJ, Hill CS Jr, Thorpe DM, Merrill JM. Physicians' attitudes toward pain and the use of opioid analgesics: Results of a survey from the Texas Cancer Pain Initiative. South Med / 2000; 93:479-487.

6. Rich BA. A legacy of silence: Bioethics and the culture of pain. J Med Humanit 1997; 18:233-259.

7. American College of Physicians ahCoME. American College of Physicians Ethics Manual, Part I: History of medical ethics, the physician and the patient, the physician's relationship to other physicians, the physician and society. Annals of Internal Medicine 1984; 101:129-137.

8. Ferrell B, Cronin N, Warfield C. The role of patient-controlled analgesia in the management of cancer pain. J Pain Sympt Manage 1992; 7:149-154.

9. Hoffmann DE. Pain management and palliative care in the era of managed care: Issues for health insurers. J Law Med Ethics 1998; 26:267-289.

10. Cleeland CS. Strategies for improving cancer pain management. J Pain Symp- tom Manage 1993; 8:361-364.

11. Grossman SA, Sheidler VR, Swedeen K, Mucenski J, Piantadosi S. Correlation of patient and caregiver ratings of cancer pain. J Pain Symptom Manage 1991; 6:53-57.

12. Proulx K, Jacelon C. Dying with dignity: The good patient versus the good death. Am J Hosp Palliat Care 2004; 21:116-120.

13. Moseley L. Unraveling the barriers to reconceptualization of the problem in chronic pain: The actual and perceived ability of patients and health professionals to understand the neurophysiology. The Journal of Pain 2003; 4:184189.

14. Dar R, Beach CM, Barden PL, Cleeland CS. Cancer pain in the marital system: A study of patients and their spouses. I Pain Symptom Manage 1992; 7:87-93.

15. Rich BA. An ethical analysis of the barriers to effective pain management. Cambridge Quarterly of Healthcare Ethics 2000; 9:54-70.

16. Peppin JF. (Bioethics and pain. In: Boswell MV \& Cole BE (eds). Weiner's Pain Management: A Practical Guide for Clinicians, Seventh Edition. CRC Press, Taylor \& Francis Group, Boca Raton, Florida, 2005; 1377-1392.

17. Merriam Webster On Line Dictionary, http://12.129.203.38/dictionary/marginalization

18. Giordano J. The good patient: Responsibilities and obligations of the patientphysician relationship. Prac Pain Management 2007; 7:58-65.

19. Wurzman R, Jonas W, Giordano J. Pain and depression: Potential and possi- bility for putative spectrum disorders. Pain Practitioner 2008; 17(3).

20. Green CR, Wheeler JRC, Marchant B, LaPorte F, Guerrero E. Analysis of the physician variable in pain management Pain Medicine 2001; 2:317-327

21. Morrison RS, Magaziner J, McLaughlin MA, Orosz G, Silberzweig SB, Koval KJ, Siu AL. The impact of post-operative pain on outcomes following hip fracture. Pain 2003; 103:303-311.

22. Lynch EP, Lazor MA, Gellis JE, Orav J, Goldman L, Marcantonio ER. The impact of postoperative pain on the development of postoperative delirium. Anesth Analg 1998; 86:781-785.

23. Perry SW. Pain on a burn unit. General Hospital Psychiatry 1984; 6:308-316.

24. Perry SW. Irrational attitudes toward addicts and narcotics. Bulletin New York Academy Medicine 1985; 61:706727.

25. http://meld.medbiq.org/meld _library/healthcare_ed_orgs/currmit_ intro_salas.htm

26. Weinstein SM, Laux LF, Thornby JI, Lorimor RJ, Hill CS, Thorpe DM, Merrill JM. Medical Students' attitudes toward pain and the use of opioid analgesics: implications for changing medical school curriculum, South Med J 2000; 93:472-478.

27. Flexner, A. Medical education in the United States and Canada: A report to the Carnegie Foundation for the Advancement of Teaching. Bulletin 4. Carnegie Foundation for the Advancement of Teaching, New York, New York, 1910. 